\section{THE RELATIONSHIP BETWEEN IL-10, IL-17, IL-23 AND VITAMIN D LEVELS, AND DISEASE ACTIVITY OF SYSTEMIC LUPUS ERYTHEMATOSUS}

${ }^{1} \mathrm{~T}$ Senturk*, ${ }^{2} \mathrm{BG}$ Cetin, ${ }^{3} \mathrm{~N}$ Aydin. ${ }^{1}$ Adnan Menderes University - Medical Faculty, Rheumatology, Aydin, Turkey; ${ }^{2}$ Adnan Menderes University - Medical Faculty, Internal Medicine, Aydin, Turkey; ${ }^{3}$ Adnan Menderes University - Medical Faculty, Medical Microbiology, Aydin, Turkey

\subsection{6/lupus-2017-000215.315}

Background and aims Several cytokines such as IL-10, IL-17, IL-23, and vitamin D have been suspected in the pathogenesis of SLE. However, the association between these cytokines, vitamin $\mathrm{D}$ and disease activity is unknown. We aimed to determine the association between IL-10, IL-17, IL-23, vitamin $\mathrm{D}$ and SLEDAI score.

Methods We included 40 patients with SLE and 20 healthy controls in the study. Clinical and laboratory parameters and, SLEDAI score were evaluated. Serum IL-10, IL-17 and IL-23 were measured by nephelometry and vitamin D by HPLC. Mann-Whitney U and Kolmogorov-Smirnov test were used for statistical analysis.

Results The level of vitamin D was significantly lower $(p=0003)$, and IL-23 was significantly higher $(p=0001)$ in SLE patients compared to healthy controls. There was no significant difference for IL-10 and IL-17 between both group $(p>0,05)$. However, a significant correlation between vitamin $\mathrm{D}$ and disease duration $(\mathrm{p}=0,02)$, and between IL-23 and vita$\min$ D $(p=0019)$ were found among SLE patients. Vitamin D levels were correlated with SLEDAI score and IL-23 in patients group.

Conclusions Although there are studies suppporting the role of IL-10 and IL-17 in the pathogenesis of SLE in the literature, there was no significant difference between patients and healthy controls in our study. IL-23 levels were significantly higher, whereas vitamin D levels were significantly lower in SLE patients than in the control group. Also vitamin D levels were negative correlated with duration of disease and IL-23. Levels of IL-23 may be used to evaluated the disease activity, or may be a promising therapeutic approach for SLE patients.

\section{THE LEVEL OF CD64 EXPRESSION ON MONOCYTE CORRELATES WITH THE ACTIVITY OF LUPUS NEPHRITIS}

${ }^{1} \mathrm{~T}$ Shimizu*, $\mathrm{S}$ Tsuji, ${ }^{2} \mathrm{~A}$ Kikuchi-Taura, ${ }^{1} \mathrm{E}$ Oguro, ${ }^{1} \mathrm{Y}$ Okita, ${ }^{1} \mathrm{M}$ Shigesaka, ${ }^{1} \mathrm{H}$ Matsuoka, ${ }^{1} \mathrm{~T}$ Nii, ${ }^{1} \mathrm{~S}$ Teshigawara, ${ }^{1} \mathrm{E}$ Kudo-Tanaka, ${ }^{1} \mathrm{Y}$ Harada, ${ }^{1} \mathrm{M}$ Matsushita, ${ }^{2} \mathrm{~S}$ Ohshima, 1J Hashimoto, ${ }^{2}$ Y Saeki. 'Osaka Minami Medical Centre, Rheumatology and Allergology, Kawachinagano, Japan; ${ }^{2}$ Osaka Minami Medical Centre, Clinical Research, Kawachinagano, Japan

\subsection{6/lupus-2017-000215.316}

Background and aims Lupus nephritis (LN) is one of the most serious clinical manifestations of systemic lupus erythematosus (SLE). Recently, we have reported that the expression levels of CD64 on monocyte (mCD64), a high-affinity receptor for IgG (FcgRI), correlate with the disease activity of SLE (Lupus 2015;24:1076-80). However, the relation between lupus nephritis (LN) and mCD64 expression is yet to be elucidated. The aim of this study is to investigate whether or not mCD64 expression level correlates with the activity of LN.

Methods We quantitatively measured the mCD64 expression levels by flow cytometry in eight SLE patients with biopsy proven LN before and after treatment. All patients fulfilled

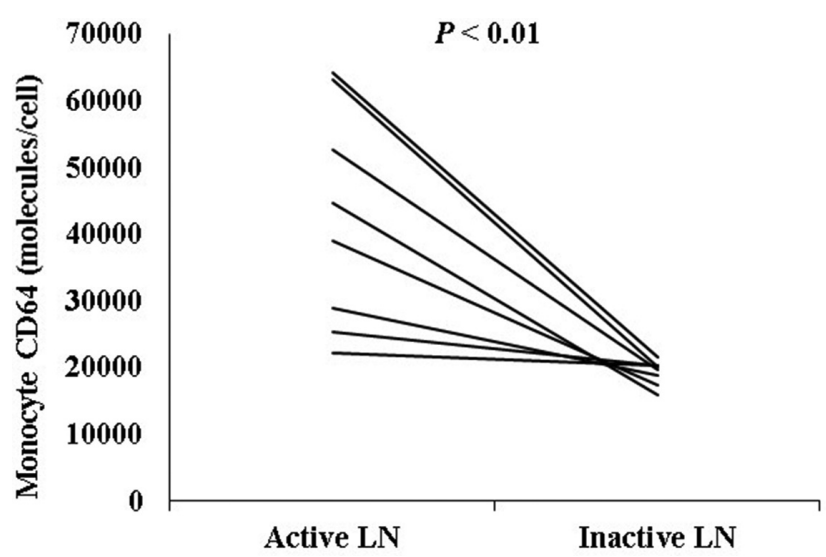

Abstract 316 Figure 1

the 1997 American College of Rheumatology classification criteria for SLE. The mCD64 expression levels of the individual patients were measured both at active (presence of proteinuria $>0.5 \mathrm{~g} /$ day and/or active urinary sediment) and inactive phase (absence of proteinuria and active urinary sediment). The changes were analysed statistically (Wilcoxon signed-rank test). Results The mean \pm SD of mCD64 expression levels before and after treatment were $42463 \pm 15466$ and $19190 \pm 1696$ moleecules/cell, respectively $(\mathrm{p}<0.01$, Wilcoxon signed-rank test). The mCD64 expression levels in active LN was significantly higher than in inactive LN.

Conclusions The mCD64 expression level correlates with the activity of LN, although a larger scale study is needed to confirm the results.

\section{DYSREGULATION OF MIRNAS EXPRESSION LEVELS AND DISEASE ACTIVITY IN SLE PATIENTS}

${ }^{1} \mathrm{R}$ Shumnalieva, ${ }^{2} \mathrm{D}$ Kachakova, ${ }^{1} \mathrm{~S}$ Monov, ${ }^{2} \mathrm{R}$ Kaneva, ${ }^{3} \mathrm{~V}$ Choumnalieva-Ivanova, 'Z Kolarov*, ${ }^{1} \mathrm{R}$ Rashkov. ${ }^{1}$ Clinic of Rheumatology, Department of Internal MedicineMedical University - Sofia, Sofia, Bulgaria; ${ }^{2}$ Molecular Medicine Centre, Department of Chemistry and Biochemistry- Medical University - Sofia, Sofia, Bulgaria; ${ }^{3}$ Alexandrovska Hospital, Department of Ophthalmology- Medical University - Sofia, Sofia, Bulgaria

\subsection{6/lupus-2017-000215.317}

Background and aims Altered expression of miRNAs have been implicated in the pathogenesis of SLE due to their role in both the adaptive and innate immunity. miRNAs control the differentiation and immunological functions of $\mathrm{B}$ cells, induction pathways in $\mathrm{T}$ cells, activation, function and maintenance of regulatory T-cells. The aim of our study was to evaluate the peripheral blood (PB) expression of miRNAs in SLE patients and to determine their correlation with the disease activity (DA).

Methods 40 SLE patients were included in the study. miR146a and miR-155 expression levels in whole PB samples were determined by PCR (SYBR Green technology). $2^{-\Delta \Delta \mathrm{Ct}}$ method was used for analysis. 32 healthy donors were used as controls. The DA was determined by DA index (SLEDAI) with 24 descriptors.

Results miR-146a was overexpressed in $62.5 \%$ of the patients. None of the patients showed underexpression of miR-146a. miR-155 was overexpressed in 50\% and underexpressed in 